\title{
Spectroscopic investigation of stars on the lower main sequence $\star, \star \star$
}

\author{
T. V. Mishenina ${ }^{1,2}$, C. Soubiran ${ }^{1}$, O. Bienaymé ${ }^{3}$, S. A. Korotin ${ }^{2}$, S. I. Belik ${ }^{2}$, I. A. Usenko², and V. V. Kovtyukh ${ }^{2}$ \\ ${ }^{1}$ Université de Bordeaux - CNRS - Laboratoire d'Astrophysique de Bordeaux, BP 89, 33271 Floirac Cedex, France \\ e-mail: Caroline. Soubiran@obs.u-bordeaux1.fr \\ 2 Astronomical Observatory, Odessa National University T.G. Shevchenko Park, Odessa 65014, Ukraine \\ e-mail: tamar@deneb1.odessa.ua; serkor@skyline.od.ua \\ 3 Observatoire Astronomique de l'Université Louis Pasteur, 11 rue de l'Université, Strasbourg, France \\ e-mail: bienayme@astro.u-strasbg.fr
}

Received 10 June 2008 / Accepted 9 July 2008

ABSTRACT

\begin{abstract}
Aims. The aim of this paper is to provide fundamental parameters and abundances with a high accuracy for a large sample of cool main sequence stars. This study is part of wider project, in which the metallicity distribution of the local thin disc is investigated from a complete sample of $\mathrm{G}$ and $\mathrm{K}$ dwarfs within $25 \mathrm{pc}$.

Methods. The stars were observed at high resolution and a high signal-to-noise ratio with the ELODIE echelle spectrograph. The $V \sin i$ were obtained with a calibration of the cross-correlation function. Effective temperatures were estimated by the line depth ratio method. Surface gravities $(\log g)$ were determined by two methods: parallaxes and ionization balance of iron. The $\mathrm{Mg}$ and $\mathrm{Na}$ abundances were derived using a non-LTE approximation. Abundances of other elements were obtained by measuring equivalent widths.

Results. Rotational velocities, atmospheric parameters $\left(T_{\text {eff }}, \log g,[\mathrm{Fe} / \mathrm{H}], V_{\mathrm{t}}\right)$, and $\mathrm{Li}, \mathrm{O}, \mathrm{Na}, \mathrm{Mg}, \mathrm{Al}, \mathrm{Si}, \mathrm{Ca}, \mathrm{Sc}, \mathrm{Ti}, \mathrm{V}, \mathrm{Cr}, \mathrm{Co}, \mathrm{Ni}$, and $\mathrm{Zn}$ abundances are provided for 131 stars. Among them, more than 30 stars are active stars with a fraction of BY Dra and RS CVn type stars for which spectral peculiarities were investigated. We find the mean abundances of the majority of elements in active and nonactive stars to be similar, except for $\mathrm{Li}$, and possibly for $\mathrm{Zn}$ and Co. The lithium is reliably detected in 54\% of active stars but only in $20 \%$ of nonactive stars. No correlation is found between $\mathrm{Li}$ abundances and rotational velocities. A possible anticorrelation of $\log A(\mathrm{Li})$ with the index of chromospheric activity GrandS is observed.

Conclusions. Active and nonactive cool dwarfs show similar dependencies of most elemental ratios vs. [Fe/H]. This allows us to use such abundance ratios to study the chemical and dynamical evolution of the Galaxy. Among active stars, no clear correlation has been found between different indicators of activity for our sample stars.
\end{abstract}

Key words. stars: fundamental parameters - stars: abundances - stars: late-type

\section{Introduction}

With this new contribution, we continue our effort to determine homogeneous atmospheric parameters and abundances for large samples of stars, dwarfs, and giants (Mishenina et al. 2004, 2006), in order to investigate the chemical and dynamical evolution of the Galactic disc. In Mishenina et al. (2004) we study the transition between the thin and thick discs in kinematics and abundance trends from nearly 200 dwarfs. In Mishenina et al. (2006), atmospheric parameters and detailed abundances of nine chemical elements were given for 177 clump giants with comparisons to theoritical predictions of stellar evolution. These data were also used to investigate the vertical distribution of the Galactic disc, with the measurement of its surface mass density (Bienaymé et al. 2006) and its AMR and AVR (Soubiran et al. 2008), and for the construction of a model of chemical evolution (Nikityuk \& Mishenina 2006).

\footnotetext{
* Based on spectra collected with the ELODIE spectrograph at the $1.93-\mathrm{m}$ telescope of the Observatoire de Haute Provence (France).

$\star \star$ Tables A.1-A3 are only available in electronic form at the CDS via anonymous ftp to cdsarc.u-strasbg.fr $(130.79 .128 .5)$ or via http://cdsweb.u-strasbg.fr/cgi-bin/qcat?J/A+A/489/923
}

Following this approach, in which kinematical and chemical information is combined, long-lived $\mathrm{G}$ and $\mathrm{K}$ dwarfs within 25 pc from the Sun were selected in the Hipparcos catalogue in order to investigate the metallicity distribution in the Solar Neighbourhood from an unbiased sample and with accurate spectroscopic metallicities. Investigation of this part of the main sequence is also interesting because it is occupied by a variety of stars showing emission in their spectra. Among the 131 stars studied here, more than 30 are variable stars belonging to the class of flaring stars, and, basically, to the subclass BY Dra, spotted stars with chromospheric activity. Up to now, no investigation of chemical composition was made for a large sample of such stars. In general, spectral research on these stars is related to the analysis of spots and spot activity on their surface. Thus, the sample of $\mathrm{G}$ and $\mathrm{K}$ dwarfs presented here gave us an unique opportunity to analyse the spectral features and chemical composition of these active stars in comparison to less active dwarfs. This is also of particular interest because the Sun is suspected of belonging to the BY Dra type. In this connection, it is obviously important to accurately determine atmospheric parameters and chemical composition and to investigate 1) the presence of peculiarities in the spectra of active stars; 2) the dependence of 
various properties upon star rotation $(v \sin i)$ and chromospheric activity; 3) the general behaviour of various element's abundances with metallicity $[\mathrm{Fe} / \mathrm{H}]$ of active and nonactive stars; and 4) the lithium abundance as an indicator of chromospheric activity.

\section{Observations and spectral processing}

Our target stars were selected in the Hipparcos catalogue with the following criteria:

$0.4 \leq(B-V) \leq 1.2$

$\pi \geq 40$ mas

$\delta \geq 0^{\circ}$

$M_{V}>4$

where $(B-V)$ is the Johnson colour deduced from the Tycho-2 photometry (Høg et al. 2000), $\pi$ the Hipparcos parallax, $\delta$ the ICRS declination, and $M_{V}$ the visual absolute magnitude deduced from $\pi$ and $V$. After elimination of 14 binaries, this selection includes 312 stars among which those lacking previous spectroscopic analysis were chosen to be observed at a highresolution, high signal-to-noise ratio $(\mathrm{S} / \mathrm{N})$.

The spectra of 131 stars were obtained using the $1.93 \mathrm{~m}$ telescope at Observatoire de Haute-Provence (OHP, France) equipped with the échelle-spectrograph ELODIE which gives a resolving power of $R=42000$. Although ELODIE spectra cover a wide range, we have only used the spectral region from $4400 \AA$ to $6800 \AA$ where the $\mathrm{S} / \mathrm{N}$ is the highest. The $\mathrm{S} / \mathrm{N}$ of the spectra range from 130 to 230 at $5500 \AA$. The extraction of the $1 \mathrm{D}$ spectra and measurement of radial velocities were performed with the standard on-line reduction software, while the deblazing and cosmic particles removal were carried out following Katz et al. (1998). The further processing of spectra (continuum level location and measurement of the equivalent widths) was performed using the software package DECH20 (Galazutdinov 1992). The equivalent widths were measured by a Gaussian fitting.

\section{Peculiarities of the targets}

The position of our target stars in the H-R diagram is given in Fig. 1. Bolometric magnitudes $M_{\text {bol }}$ were determined by the relation $M_{\mathrm{bol}}=M_{V}+\mathrm{BC}$, where the bolometric corrections $\mathrm{BC}$ were obtained by interpolating the values tabulated by Flower (1996).

According to Simbad, there are more than 30 stars classified as variable or active stars in our sample. The wide class of flare (Fl) stars (generally UV Cet type) is divided into subclasses, including the spotted short-amplitude stars of BY Dra type (Chugainov 1966) with spectral types $\mathrm{F}$ to $\mathrm{M} \mathrm{V}$ and $v \sin i<20 \mathrm{~km} \mathrm{~s}^{-1}$, and the RS CVn type stars which are detached or semi-detached systems with components $F$ to G V-IV and $\mathrm{G}$ to $\mathrm{K}$ IV. Flare stars have masses ranging from 0.05 to $1.5 \mathrm{M}_{\odot}$, ages from $10^{6}$ to $10^{9}$ years, and periods of axial rotation from about $10 \mathrm{~h}$ to about 10 days. The BY Dra type stars exhibit differential rotation (equator rotates faster than poles) and cycles of activity (similar to the 11 years cycle of the Sun, Gershberg 2002).

Previous photometric and spectral research on active stars has been mainly directed to the study of temperatures and distribution of spots. Some success have been achieved with Doppler

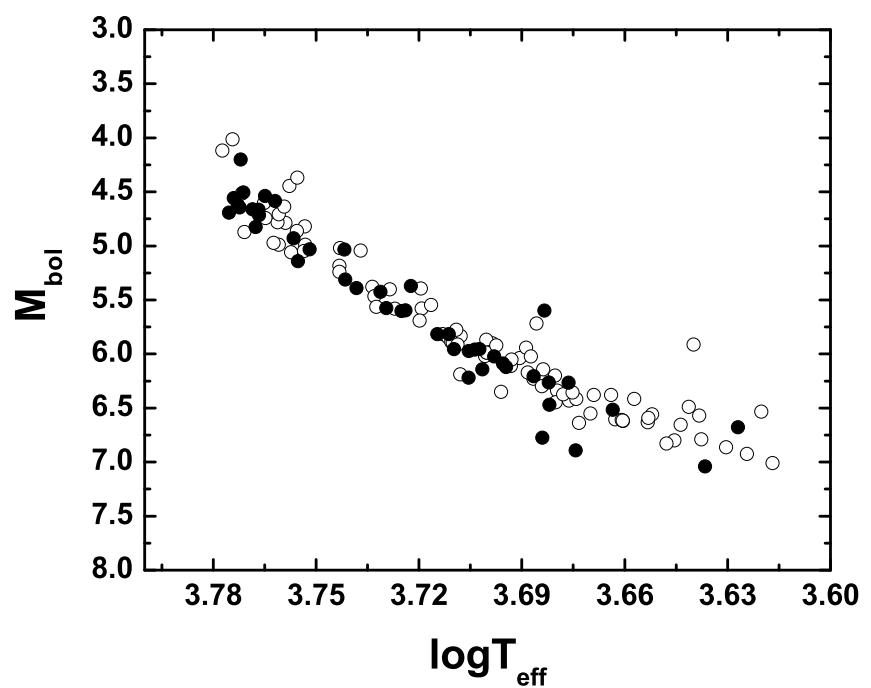

Fig. 1. Location in the H-R diagram of nonactive (open circles) and active (black circles) stars studied in this work.

imaging and Zeeman spectroscopy at high resolution (Alekseev 2006).

Our list includes some known stars of BY Dra type: V439 And, V435 And, V538 Aur, OU Gem, DX Lyn, HP Boo, V1654 Aql, V1803 Cyg, HN Peg, V453 And, V833 Tau; and of RS CVn type SV LMi, V368 Cep and V774 Her (Fl). We found that some active stars on our list show appreciable $\mathrm{H}_{\alpha}$ and $\mathrm{H}_{\beta}$ emissions. There are OU Gem, V775 Her, V833 Tau, V430 Cep (BY Dra type), and RS CVn-V368 Cep, but also in other flaring stars we found the change in these lines cores, but to a much smaller degree.

There are 6 stars simply classified in Simbad as variable stars: HD 20630 ( $\kappa$ Cet), HD 25680 (NSV 1452), HD 26923 (V774 Tau), HD 190406 (NSV 12757), HD 217813 (MT Peg). According to Biazzo et al. (2007), Hall et al. (2007), Lyra \& Porto de Mello (2005), and Wright et al. (2004), all show chromospheric activity in the $\mathrm{Ca}$ II line and $\mathrm{H}_{\alpha}$. Moreover, the stars HD 20630 and HD 205434 are classified as BY Dra type in the "The 74th Special Name-list of Variable Stars" by Kazarovets et al. (1999). Biazzo et al. (2007) have modelled the distribution of spots for HD 20630. All these stars define a group of active stars with chromospheric activity that will be compared in the following to the rest of the sample supposed to be made of nonactive stars.

The comparison of the spectra of variable stars and usual dwarfs with comparable atmospheric parameters outside of the regions of $\mathrm{H}_{\alpha}$ and $\mathrm{H}_{\beta}$ do not show any difference. The absence of obvious discrepancy has allowed us to assume that we can apply to them standard methods of investigation.

\section{Rotational velocities}

We measured the rotational velocities $(v \sin i)$ of all our targets with a relation, calibrated by Queloz et al. (1998), giving $v \sin i$ as a function of $\sigma_{\mathrm{RV}}$, the standard deviation of the ELODIE cross-correlation function approximated by a Gaussian. The relation is

$v \sin i=1.90 \sqrt{\sigma_{\mathrm{RV}}^{2}-\sigma_{0}^{2}}$. 
Table 1. Comparaison of our $v \sin i$ determinations with those from other authors (mean difference, standard deviation, and number of common stars).

\begin{tabular}{rcl}
\hline \hline$\Delta v \sin i$ & $n$ & source \\
\hline$-0.2 \pm 1.3$ & 91 & Nordström et al. (2004) \\
$-0.4 \pm 1.3$ & 58 & Valenti \& Fisher (2005) \\
$-0.6 \pm 1.9$ & 21 & Tokovinin (1992) \\
$0.2 \pm 1.0$ & 10 & Benz \& Mayor (1984) \\
$-0.5 \pm 0.7$ & 10 & Gaidos et al. (2000) \\
\hline
\end{tabular}

The parameter $\sigma_{0}$ represents the mean intrinsic width for nonrotating stars. It has been calibrated by Queloz et al. (1998) as a function of $(B-V)$ :

$\sigma_{0}=0.27(B-V)^{2}+4.51$.

This $v \sin i$ calibration is valid for stars in the colour range $0.7 \leq$ $B-V \leq 1.4$, with slow and moderate rotation rates $\left(\leq 40 \mathrm{~km} \mathrm{~s}^{-1}\right)$ and solar metallicities, which is consistent with the majority of our stars. The 5 metal-poor stars with $-0.6 \leq[\mathrm{Fe} / \mathrm{H}] \leq-0.4$ may have $v \sin i$ determinations that are slightly underestimated, while it is the contrary for the 3 super metal-rich stars of the sample. The typical precision of the $v \sin i$ determinations with this method is $1 \mathrm{~km} \mathrm{~s}^{-1}$.

The comparison of our $v \sin i$ determinations with 5 other studies is shown in Table 1. The agreement is good. Rotational velocities are given in Table A.1.

\section{Atmospheric parameters}

Variable stars of BY Dra type have, basically, insignificant fluctuations of their magnitudes, variations in $(B-V)$ do not exceed $0.1 \mathrm{mag}$. However, some variable stars may have variations in $(B-V)$ up to $0.4 \mathrm{mag}$. Two of them, V833 Tau (HD 283750) and V775 Her (HD 175742), are the part of our investigation. In such cases, the use of photometric calibrations to determine $T_{\text {eff }}$ may lead to significant errors in temperature values; for instance, errors of 0.02 and 0.10 in $(B-V)$ give errors in $T_{\text {eff }}$ of 40 and $170 \mathrm{~K}$, respectively. In our work, effective temperatures $T_{\text {eff }}$ were estimated by the line depth ratio method developed by Kovtyukh et al. (2004), which provides a typical precision better than $10 \mathrm{~K}$. This method is based on the calibration of the ratio of the central depths (or equivalent widths) of two lines having very different functional $T_{\text {eff }}$ dependences. It is independent of interstellar reddening and is not influenced much by the individual properties of the stars, such as rotation, microturbulence, and metallicity. These effects, as well as NLTE effects and the effects of individual chemical composition, can be reduced by the statistics of a large number of different line ratios. For a given star, the obtained temperature characterises the conditions of its atmosphere at the epoch of observation, which is very important in the case of variable stars.

The surface gravities $\log g$ were determined by two methods for the 80 stars with effective temperatures higher than $5000 \mathrm{~K}$ (ionization balance of iron and using parallaxes), showing an average difference of $\left\langle\log g_{\mathrm{IE}}-\log g_{\mathrm{P}}\right\rangle=-0.06 \pm 0.16$. For cooler stars, which lack Fe II lines, only the parallax method was used. The microturbulent velocity $V_{\mathrm{t}}$ was determined by forcing all the FeI lines to give the same iron abundances regardless of EW. The adopted metallicity $[\mathrm{Fe} / \mathrm{H}]$ is the iron abundance determined from $\mathrm{Fe}$ I lines.

In Table 2, we compare our determinations of atmospheric parameters with those of other authors. There is no significant
Table 2. Comparaison of our atmospheric parameters with those from other authors.

\begin{tabular}{|c|c|c|c|}
\hline$\Delta T_{\text {eff }}$ & $\Delta[\mathrm{Fe} / \mathrm{H}]$ & $n$ & source \\
\hline $19 \pm 70$ & & 29 & Masana et al. (2006) \\
\hline$-7 \pm 62$ & $-0.12 \pm 0.15-0.03 \pm 0.07$ & 61 & Valenti \& Fisher (2005) \\
\hline $26 \pm 26$ & $-0.04 \pm 0.15 \quad 0.01 \pm 0.04$ & 22 & Fuhrmann (2008) \\
\hline $1 \pm 73$ & $-0.01 \pm 0.17-0.03 \pm 0.04$ & 14 & Gaidos \& Gonzalez (2002) \\
\hline$-7 \pm$ & $16 \quad 0.0$ & 17 & Takeda et al. $(2005,2007)$ \\
\hline
\end{tabular}

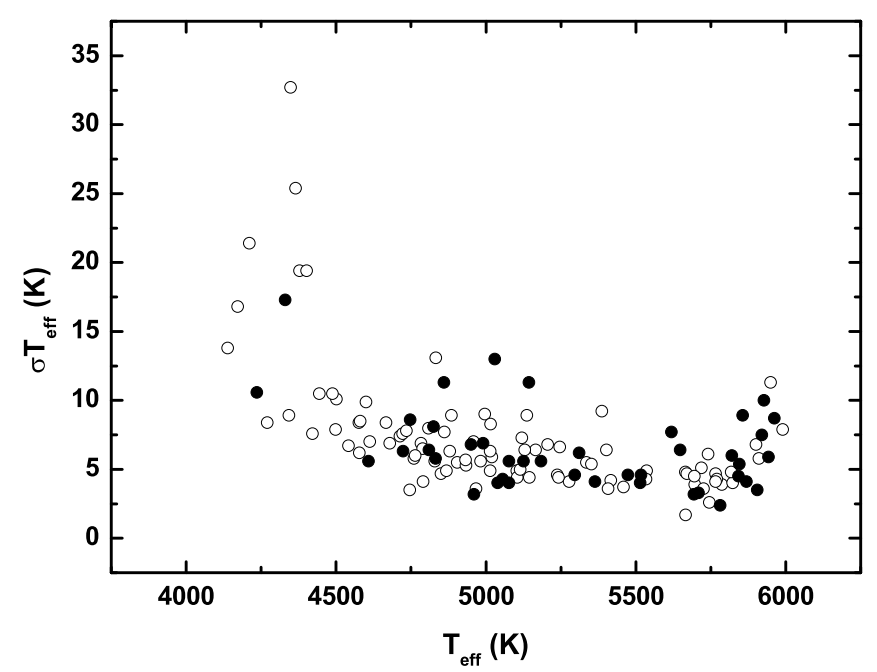

Fig. 2. Errors on temperature, $\sigma T_{\text {eff }}$, vs. $T_{\text {eff. Notation is the same as in }}$ Fig. 1.

difference. We note the excellent agreement of our scales with those of Fuhrmann (2008). The errors of our temperature determinations are given for each star as a product of the line depth ratio method. For $\log g, V_{\mathrm{t}}$, and $[\mathrm{Fe} / \mathrm{H}]$, we estimate our error bars to respectively be $0.2 \mathrm{dex}, 0.2 \mathrm{dex}$, and $0.05 \mathrm{dex}$.

To investigate the precision of our $T_{\text {eff }}$ determinations and the influence of variability, we constructed the dependence of $\sigma T_{\text {eff }}$ on $T_{\text {eff }}$ and $V$ magnitude for the active and nonactive stars (Figs. 2 and 3). As can be seen, the scatter is generally below $10 \mathrm{~K}$ but is increasing up to $30 \mathrm{~K}$ at low temperatures $\left(T_{\text {eff }}<4400 \mathrm{~K}\right)$ and for faint stars $(V<9)$; i.e. it is due to the blending of lines and to low values of $\mathrm{S} / \mathrm{N}$. Active and nonactive stars do not show any distinction. Figures 4 and 5 show the microturbulent velocity $V_{\mathrm{t}}$ and rotational velocity $v \sin i$ versus effective temperature $T_{\text {eff }}$. Among the five stars with the highest microturbulent velocity, four are active stars and one, HD 213245, is not part of our sample of active stars, but could be active since its index of chromospheric activity $S$ measured by Wright et al. (2004) has a high value $(S=0.602)$. For the other stars, with lower microturbulent velocity, there is no distinction between the 2 groups of active and nonactive stars. Among the ten stars with $v \sin i>6 \mathrm{~km} \mathrm{~s}^{-1}$, all are active stars, except one, HD 97658, for which not much can be said about its activity. It has a moderate value its index of chromospheric activity $(S=0.395)$. For the other stars with $v \sin i<6 \mathrm{~km} \mathrm{~s}^{-1}$, no clear trend is observed. The mean rotational velocity of active stars is $3.23 \pm 1.73$, whereas it is $1.99 \pm 1.36$ for the nonactive stars. The difference is thus within the error bars.

Table A. 1 gives $T_{\text {eff }}$ and $\sigma T_{\text {eff }}, \log g, V_{\mathrm{t}}$, and metallicities $[\mathrm{Fe} / \mathrm{H}]$ of the 131 target stars. 


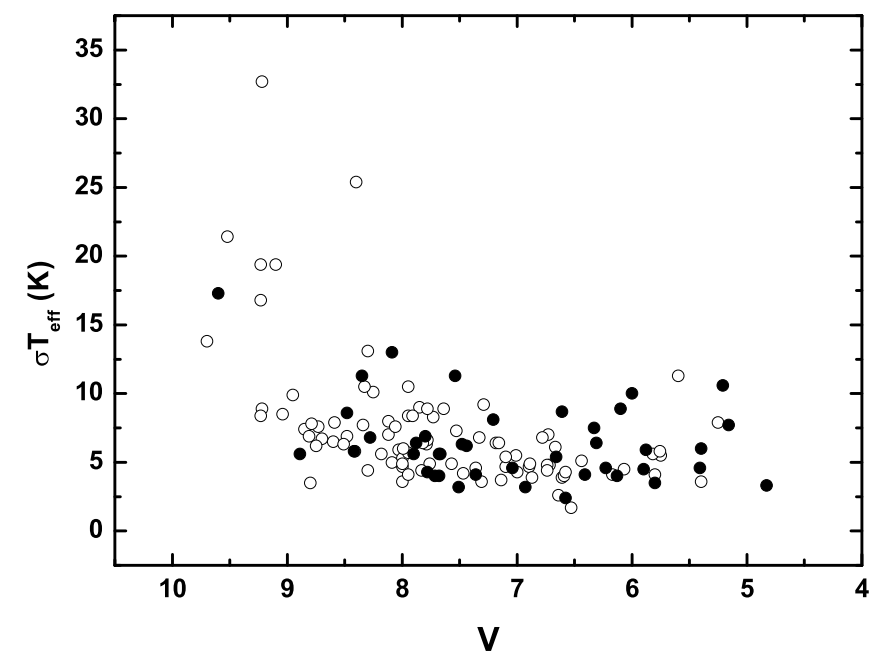

Fig. 3. Errors on temperature, $\sigma T_{\text {eff }}$, vs. $V$ magnitudes. Notation is the same as in Fig. 1.

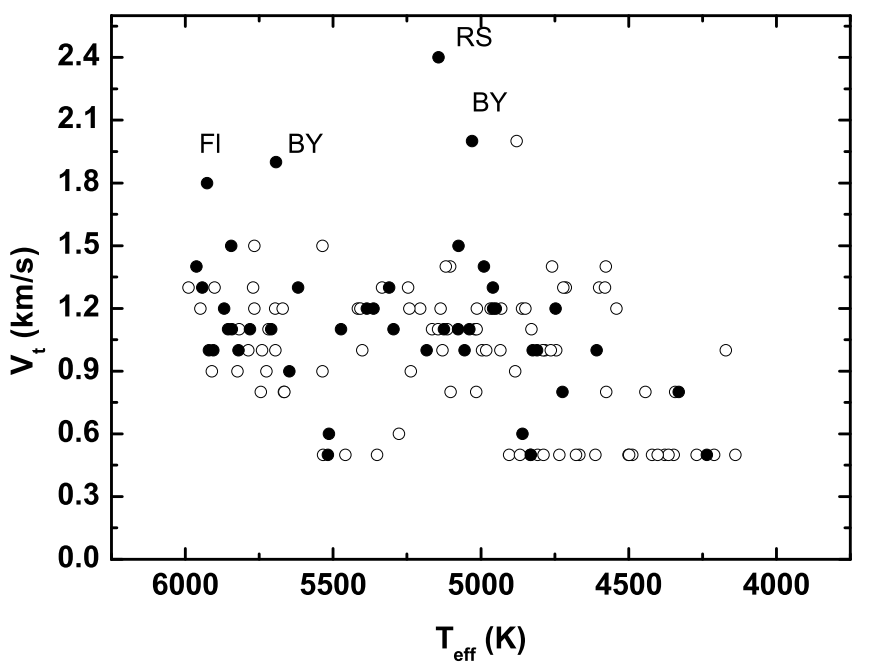

Fig. 4. Turbulent velocity $V_{\mathrm{t}}$ versus $T_{\text {eff }}$. Notation is the same as in Fig. 1.

\section{Determination of chemical composition}

We used the grid of stellar atmospheres from Kurucz (1993) to compute abundances of $\mathrm{Li}, \mathrm{O}, \mathrm{Na}, \mathrm{Mg}, \mathrm{Al}, \mathrm{Si}, \mathrm{Ca}, \mathrm{Sc}, \mathrm{Ti}, \mathrm{V}, \mathrm{Cr}$, $\mathrm{Co}, \mathrm{Ni}$, and $\mathrm{Zn}$. The choice of the model was made using a standard interpolation on $T_{\text {eff }}$ and $\log g$. The abundance analysis of $\mathrm{Al}, \mathrm{Si}, \mathrm{Ti}, \mathrm{V}, \mathrm{Cr}, \mathrm{Co}, \mathrm{Ni}$, and $\mathrm{Zn}$ was done in the LTE approximation (Kurucz's WIDTH9 code) using the measured equivalent widths of these elements' lines and the solar oscillator strengths (Kovtyukh \& Andrievsky 1999).

The elemental abundances were obtained using EW from 110 to 260 lines of Fe I, 6 to 15 lines of Fe II, 2 lines of $\mathrm{Al} \mathrm{I}$, 13 to 30 lines of Si I, 8 to 22 lines of Ca II, 8 to 9 lines of Sc II, 15 to 50 lines of Ti I, 10 to 33 lines of I, 17 to 35 lines of Cr I, 17 to 22 lines of Co I, 33 to 50 lines of Ni I, and 2 to 3 lines of $\mathrm{Zn} \mathrm{I.}$ The number of lines depends on the spectral type of the stars and the $\mathrm{S} / \mathrm{N}$ of their spectrum.

The $\mathrm{Li}$ and $\mathrm{O}$ abundances in programm stars were obtained by fitting synthetic spectra to the observational profiles. We used the STARSP LTE spectral synthesis code developed by Tsymbal (1996). Considering the wide range of temperatures and metallicities of our sample stars, special effort was put into a compilation of a full list of atomic and molecular lines close to the

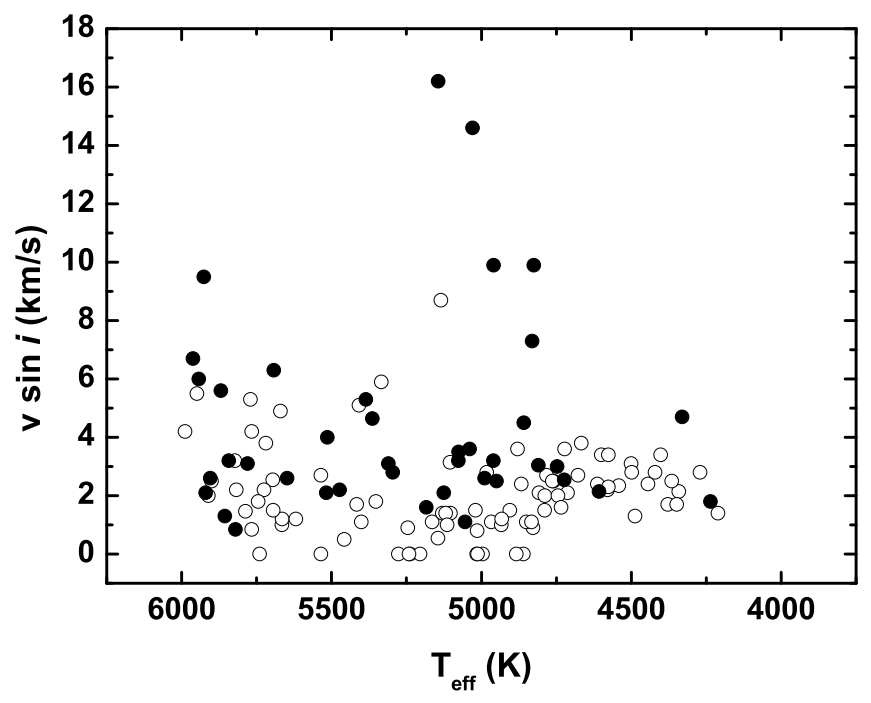

Fig. 5. Rotational velocity $v \sin i$ versus $T_{\text {eff }}$. Notation is the same as in Fig. 1.

${ }^{7} \mathrm{Li} 6707 \AA$ line (Mishenina \& Tsymbal 1997). The O abundances were determined on the [O I] $6300.3 \AA$ line by the synthetical spectrum method. The Ni I and CN blended lines were included in the final line list.

The $\mathrm{Na}$ and $\mathrm{Mg}$ abundances were determined under the NLTE assumption with the help of a modified version of the MULTI code (Carlsson 1986) by Korotin et al. (1999). In such a modified version, in particular, additional opacity sources from the ATLAS9 code (Kurucz 1993) were included. We employed the model of the $\mathrm{Mg}$ atom consisting of 97 levels: 84 levels of Mg I, 12 levels of $\mathrm{Mg}$ II, and a ground state of $\mathrm{Mg}$ III. Within the described system of the $\mathrm{Mg}$ atom levels, we considered the radiative transitions between the first 59 levels of $\mathrm{Mg}$ I and the ground level of $\mathrm{Mg}$ II. Transitions between the rest levels were not taken into account, and they were only used in the equations of particle number conservation (for details, see Mishenina et al. 2004). To determine the magnesium abundances, we used the profiles of Mg I lines $\lambda \lambda 4703,5172-83,5528$, and $5711 \AA \AA$.

The model of the sodium atom described by Sakhibullin (1987) was modified (see Korotin \& Mishenina 1999). It consists of 27 levels of $\mathrm{Na}$ I and the ground level of $\mathrm{Na}$ II. We considered the radiative transitions between the first 20 levels of $\mathrm{Na}$ I and the ground level of Na II. Transitions between the remaining levels were only used in the equations of particle number conservation. Finally, 46 bound - bound and 20 bound - free transitions were included in the lineariation procedure. For 34 transitions, the radiative rates were fixed. We used the profiles of two doublets of $\mathrm{Na}, \lambda \lambda 5682-88 \AA \AA$ and $\lambda \lambda$ 6154-6160 ̊̊.

Abundances of elements were determined from a differential analysis to the solar data. For this purpose several spectra of the Moon and asteroids were obtained and reduced in the same manner as the stellar spectra. Abundance ratios relative to $\mathrm{Fe}$ are in Table A.2.

Table 3 summarizes the total uncertainty in elemental abundance determinations due to parameter determination errors $\left(\mathrm{d} T_{\text {eff }}=+20 \mathrm{~K}, \operatorname{dlog} g=0.2, \mathrm{~d} V_{\mathrm{t}}=0.2 \mathrm{~km} \mathrm{~s}^{-1}\right)$, the choice of model metallicity $([\mathrm{Fe} / \mathrm{H}]=-0.2)$ and due to EW measurement and synthetic spectra fitting errors (in all cases we have adopted 0.03). We give the results for HD 4256. As seen from Table 3 , the total uncertainty is lower than 0.1 for all abundance determinations. 
Table 3. Uncertainties in abundance determination for HD 4256.

\begin{tabular}{lrrrrr}
\hline \hline El & $\mathrm{d} T_{\text {eff }}$ & $\mathrm{d} \log g$ & $\mathrm{~d} V_{\mathrm{t}}$ & $\mathrm{d}[\mathrm{Fe} / \mathrm{H}]$ & Total \\
\hline $\mathrm{O}$ I & -0.03 & 0.07 & 0.00 & 0.05 & 0.10 \\
$\mathrm{Na}$ I & -0.01 & 0.07 & 0.04 & 0.03 & 0.09 \\
$\mathrm{Mg}$ I & -0.01 & 0.03 & 0.02 & 0.03 & 0.05 \\
$\mathrm{Al}$ I & -0.02 & 0.04 & 0.03 & 0.01 & 0.06 \\
$\mathrm{Si} \mathrm{I}$ & 0.00 & -0.02 & 0.01 & 0.04 & 0.05 \\
$\mathrm{Ca}$ I & 0.02 & 0.05 & 0.05 & 0.03 & 0.08 \\
$\mathrm{Sc}$ II & 0.00 & -0.05 & 0.05 & 0.07 & 0.10 \\
$\mathrm{Ti} \mathrm{I}$ & -0.02 & 0.01 & 0.05 & 0.01 & 0.06 \\
$\mathrm{~V}$ I & -0.03 & 0.01 & 0.05 & 0.01 & 0.06 \\
$\mathrm{Cr}$ I & -0.02 & 0.01 & 0.03 & 0.01 & 0.06 \\
$\mathrm{Fe}$ I & -0.01 & 0.01 & 0.04 & 0.04 & 0.06 \\
$\mathrm{Fe}$ II & -0.02 & 0.05 & -0.03 & -0.07 & 0.10 \\
$\mathrm{Co}$ I & -0.01 & -0.02 & 0.05 & 0.04 & 0.07 \\
$\mathrm{Ni}$ I & 0.00 & -0.01 & 0.05 & 0.05 & 0.08 \\
$\mathrm{Zn}$ I & 0.00 & 0.04 & 0.03 & 0.08 & 0.10 \\
\hline
\end{tabular}

\section{Results and discussion}

Our sample gives us the opportunity to compare the behaviour of the elements' abundances with metallicity among active and nonactive stars. We have considered abundances ratios versus metallicity in Figs. 6 and 7, where quiet stars, and active stars. We shown computed the mean abundance values of different elements of active and nonactive stars (Table 4).

General trends in the elemental abundances correspond to earlier results on disc stars. We can observe the decrease in $\alpha$ elements with increasing metallicity, especially for $\mathrm{O}, \mathrm{Mg}, \mathrm{Si}$, and $\mathrm{Ti}$, whereas the dispersion is high for $\mathrm{Ca}$. We also get a high dispersion on $\mathrm{Al}$ that might be reduced with an NLTE analysis. The dispersion on the NLTE Na abundances is also high, as observed in many previous studies. This is often interpreted by now $\mathrm{Na}$ has many sources of production resulting in a prestellar material that is inhomogeneously enriched. In contrast, the dispersions of $\mathrm{Ni}$ and $\mathrm{V}$ are remarkably low.

As can be see from Figs. 6 and 7 the abundance behaviour of the majority of elemental abundances of active and nonactive stars does not differ. It seems that, for $\mathrm{Co}$ and $\mathrm{Zn}$, the mean abundance of active stars is lower than for the other stars; however, the difference is below the one $\sigma$ bar.

We now consider the Li abundances in the investigated stars. Tracing $\mathrm{Li}$ in different types of stellar and sub-stellar objects helps to study physical conditions and nuclear processes in their interior. Lithium is a very fragile element, which is destroyed at temperatures hotter than $2.5 \times 10^{6} \mathrm{~K}$, and this process already begins in the pre-main sequence stage. Fresh isotopes of $\mathrm{Li}$ in stellar atmospheres can be produced by nuclear interactions of ions accelerated at the surface of flare stars (Tatischeff et al. 2008). In the general case, the surface abundance of Li should be a function of stellar mass, age, metallicity, and of somewhat poorly explored physical processes like rotation, convection, mass loss rates, flares, etc. Stars of BY Dra type are young stars, with ages about $10^{8}$ years (Chugainov 1991). In their spectra, the lines of lithium, which is the indicator of activity and age of stars, are often present. However, these lines have different intensity, sometimes they are absent, and their intensity does not always correlate with other indicators of stellar activity.

We compared our determination of Li abundances with those in other papers (Takeda \& Kavanomoto 2005; Takeda et al. 2007; Luck \& Heiter 2006) in Table 5. There is a good agreement between our determinations and these studies, within error bars. For several stars we could only estimate the upper limit of the

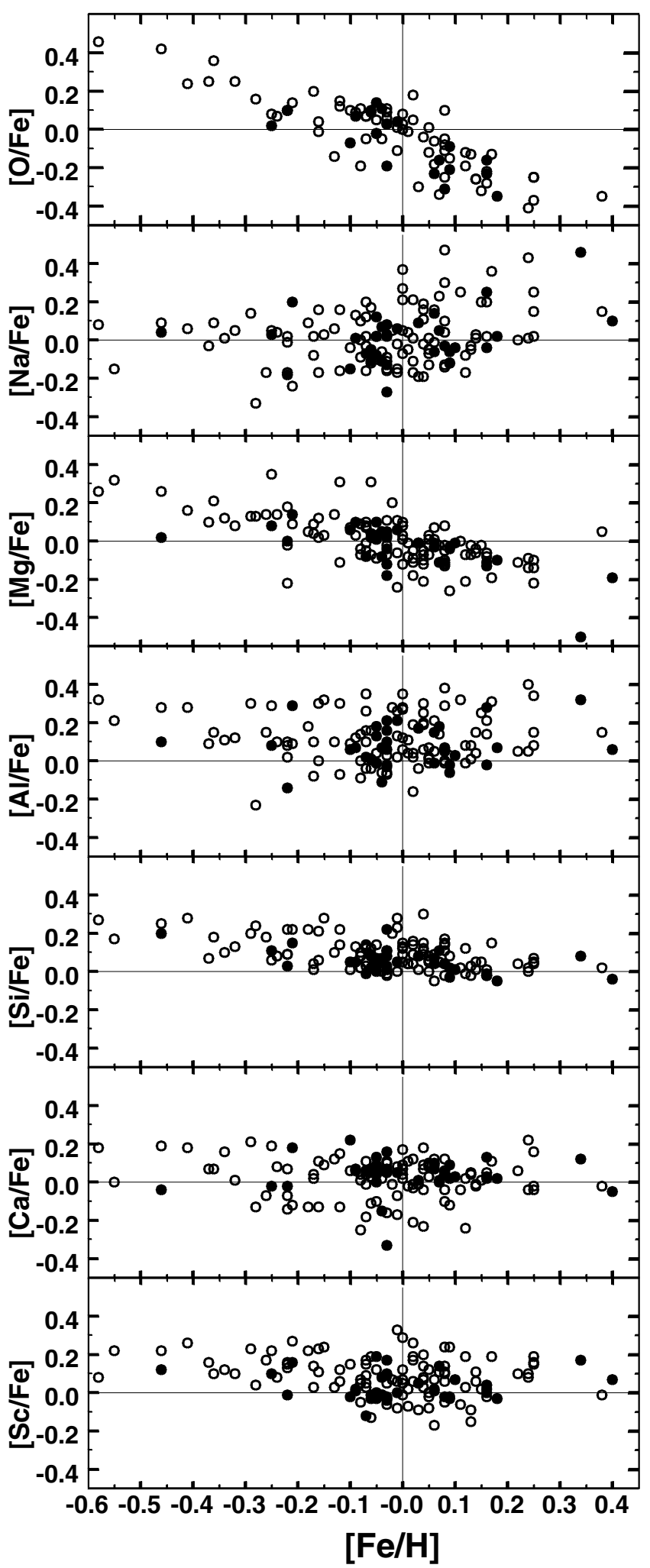

Fig. 6. Trends in the studied elements with $[\mathrm{Fe} / \mathrm{H}]$ of nonactive stars (open circles) and active (black circles) stars.

lithium abundance, and in some other cases the $\mathrm{Li}$ abundance could not be determined at all because of defects in the region of the Li lines.

The $\mathrm{Li}$ is reliably detected in 19 stars among the 91 nonactive dwarfs, while it is detected in 21 among the 39 stars with chromospheric activity, correspondonding to about $20 \%$ and $54 \%$, 


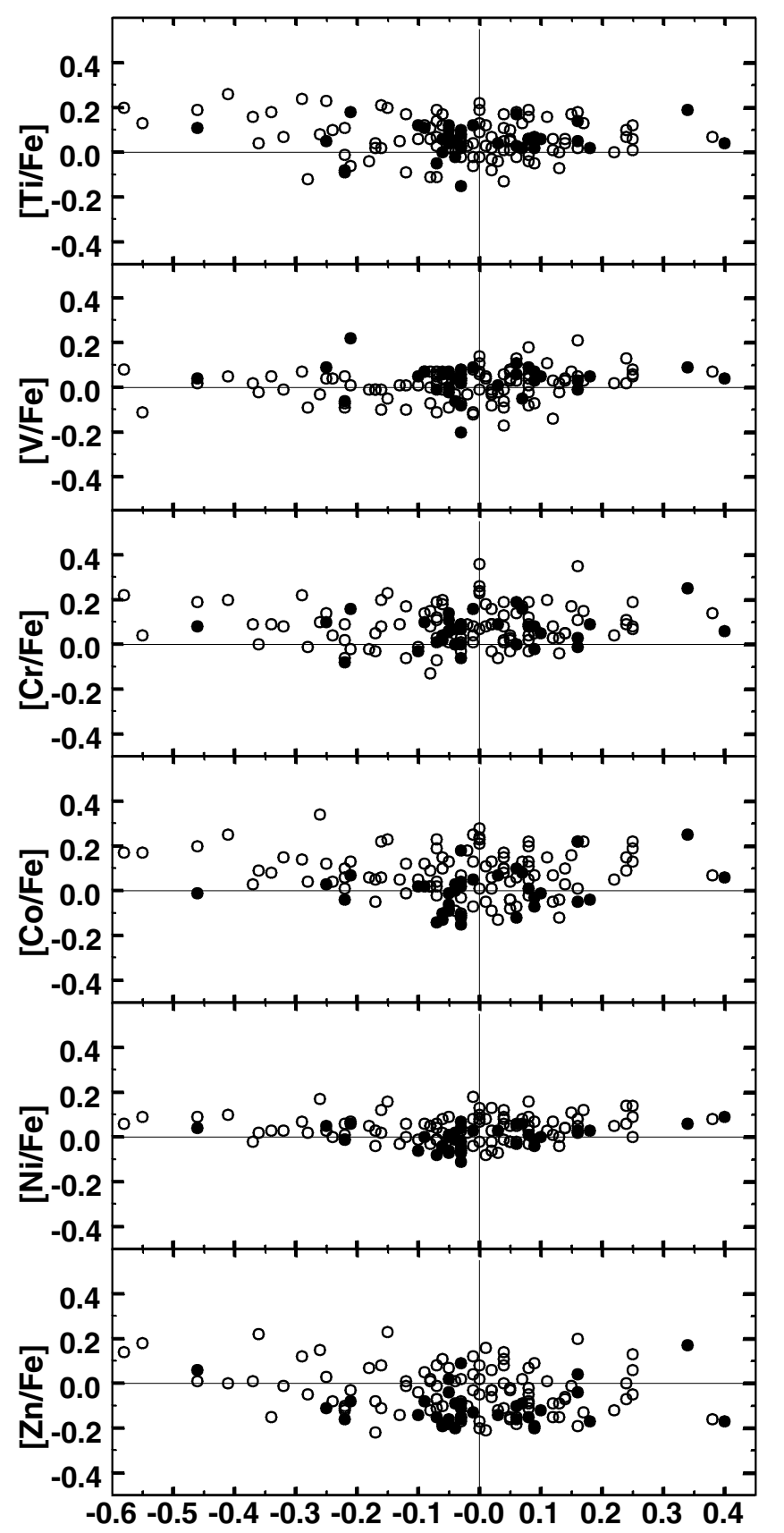

$[\mathrm{Fe} / \mathrm{H}]$

Fig. 7. Trends in the studied elements with $[\mathrm{Fe} / \mathrm{H}]$ of nonactive stars (open circles) and active (black circles) stars.

respectively. Thus the frequency of stars with Li is significantly higher in active stars than in nonactive stars.

We searched for correlations between the lithium abundance, chromospheric activity, and rotation in Figs. 8 to 10. We used as an indicator of chromospheric activity the index GrandS determined by Wright et al. (2004). The Mount Wilson "S-value", which has become a standard metric of chromospheric activity, was constructed on the ratio of the flux in the special bandpasses in the region of $\mathrm{H}$ and $\mathrm{K} \mathrm{Ca}$ II lines. The index GrandS gives some chromospheric characteristics averaged over the epochs of observations.

For active stars, no significant correlation between $\log A(\mathrm{Li})$ and $v \sin i$ can be observed (Fig. 8). We can see a large dispersion
Table 4. The mean values of elemental abundances in nonactive (NA) and active (A) stars.

\begin{tabular}{lrr}
\hline \hline Element & NA & $\mathrm{A}$ \\
\hline$\langle[\mathrm{O} / \mathrm{Fe}]\rangle$ & $-0.02 \pm 0.19$ & $-0.07 \pm 0.15$ \\
$\langle[\mathrm{Na} / \mathrm{Fe}]\rangle$ & $0.02 \pm 0.15$ & $0.01 \pm 0.13$ \\
$\langle[\mathrm{Mg} / \mathrm{Fe}]\rangle$ & $0.01 \pm 0.13$ & $-0.03 \pm 0.11$ \\
$\langle[\mathrm{Al} / \mathrm{Fe}]\rangle$ & $0.12 \pm 0.13$ & $0.08 \pm 0.11$ \\
$\langle[\mathrm{Si} / \mathrm{Fe}]\rangle$ & $0.10 \pm 0.08$ & $0.05 \pm 0.06$ \\
$\langle[\mathrm{Ca} / \mathrm{Fe}]\rangle$ & $0.02 \pm 0.10$ & $0.04 \pm 0.09$ \\
$\langle[\mathrm{Sc} / \mathrm{Fe}]\rangle$ & $0.09 \pm 0.10$ & $0.04 \pm 0.07$ \\
$\langle[\mathrm{Ti} / \mathrm{Fe}]\rangle$ & $0.06 \pm 0.09$ & $0.06 \pm 0.07$ \\
$\langle[\mathrm{~V} / \mathrm{Fe}]\rangle$ & $0.01 \pm 0.07$ & $0.03 \pm 0.07$ \\
$\langle[\mathrm{Cr} / \mathrm{Fe}]\rangle$ & $0.09 \pm 0.09$ & $0.07 \pm 0.07$ \\
$\langle[\mathrm{Co} / \mathrm{Fe}]\rangle$ & $0.08 \pm 0.10$ & $0.00 \pm 0.09$ \\
$\langle[\mathrm{Ni} / \mathrm{Fe}]\rangle$ & $0.04 \pm 0.06$ & $0.00 \pm 0.05$ \\
$\langle[\mathrm{Zn} / \mathrm{Fe}]\rangle$ & $-0.03 \pm 0.10$ & $-0.10 \pm 0.09$ \\
\hline
\end{tabular}

Table 5. Comparison of Li abundances with those from other authors.

\begin{tabular}{lll}
\hline \hline$\Delta \mathrm{Li}$ & $n$ & Source \\
\hline $0.05 \pm 0.12$ & 8 & Takeda et al. $(2005,2007)$ \\
$0.00 \pm 0.06$ & 3 & Luck \& Heiter $(2006)$ \\
\hline
\end{tabular}

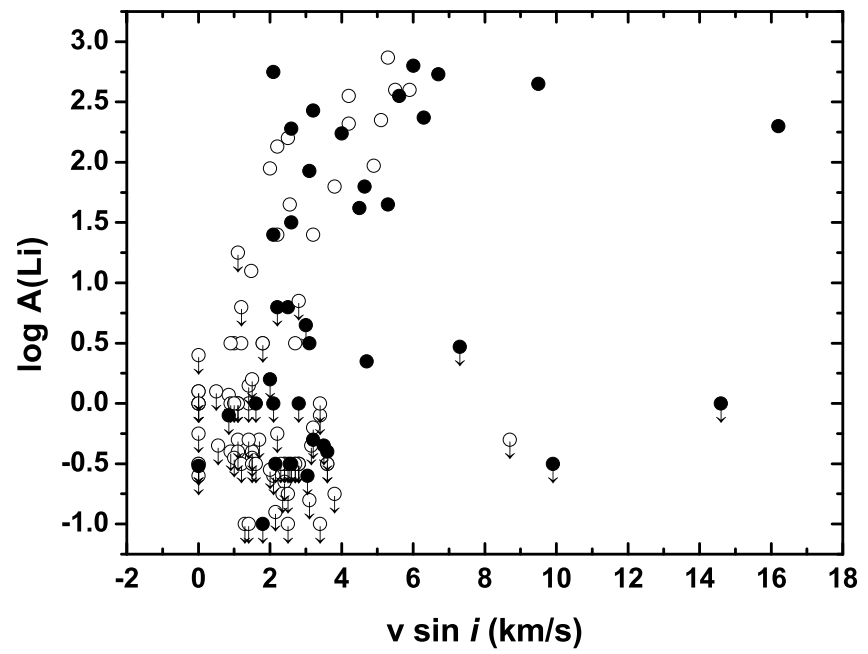

Fig. 8. The dependence of Li abundance on the rotational velocity $v \sin i$, the notation is the same as in Fig. 1. The stars with the upper limit of the $\mathrm{Li}$ abundance marked by an arrow.

of Li abundances at low $v \sin i\left(v \sin i<8 \mathrm{~km} \mathrm{~s}^{-1}\right)$. The dispersion of Li abundances at low $v \sin i$ has already been observed by Jasniewicz (2006), but for stars hotter than in our sample. Together with our study, it suggests that such a dispersion is present over a wide range of temperatures. Moreover, the dispersion is similarly high for both active and nonactive stars.

We suggest that some physical phenomena, such as dredge-up of Li by atomic diffusion for nonactive stars or different degree of nuclear events at the surface of active stars or the presence of a stellar companion, could be responsible for the high $\mathrm{Li}$ abundances in our sample. As can be seen from Fig. 9 the index of chromospheric activity GrandS has a similarly large dispersion for active and nonactive stars, and no correlation between GrandS and $v \sin i$ is visible.

Anticorrelation between the $\mathrm{Li}$ abundance $\log A(\mathrm{Li})$ and the index of chromospheric activity GrandS is more appreciable. However, this anticorrelation may be due to the dependence of $\mathrm{Li}$ abundances on temperatures: our active stars with high 
Table 6. Stars with spectral peculiarities.

\begin{tabular}{rrrrrcccr}
\hline \hline HD & Name & Sp & $v \sin i$ & $\sigma T_{\text {eff }}$ & $V_{\mathrm{t}}$ & $\log A(\mathrm{Li})$ & $\mathrm{H}_{\text {emission }}$ & Type \\
\hline 45088 & OU Gem & K0 & 9.9 & 7 & 1.2 & - & + & BY \\
175742 & V775 Her & K0 & 14.6 & 13 & 2.0 & - & + & BY \\
205434 & V430 Cep & K4 & 4.7 & 17 & 0.8 & 0.35 & + & V \\
206860 & HN Peg & G0 & 9.5 & 10 & 1.8 & 2.65 & - & Fl \\
220140 & V368 Cep & G9 & 6.2 & 11 & 2.4 & 2.30 & + & RS \\
283750 & V833 Tau & K0 & 7.3 & 6 & 0.5 & - & ++ & BY \\
\hline
\end{tabular}

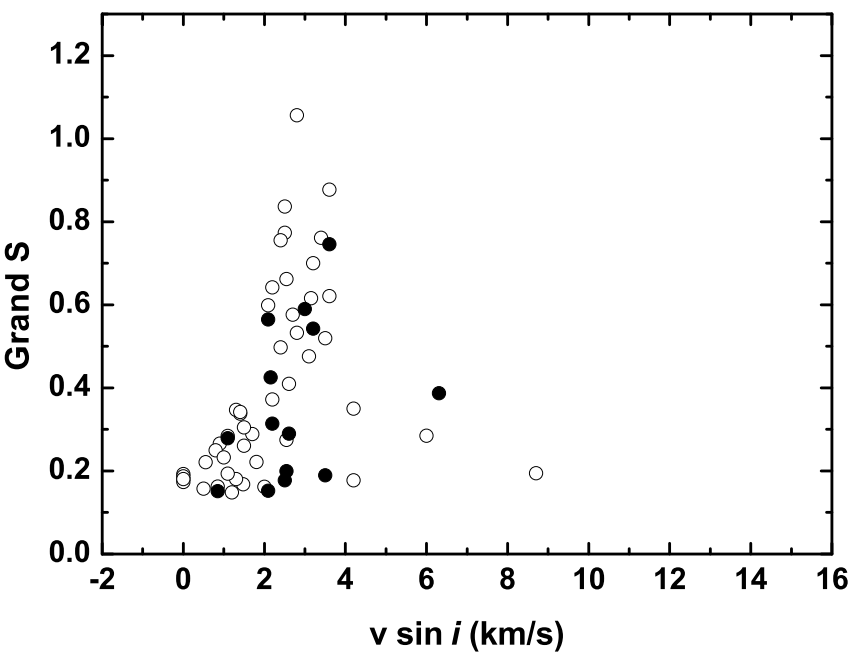

Fig. 9. Index of chromospheric activity GrandS with rotational velocity. The notation is the same as in Fig. 1.

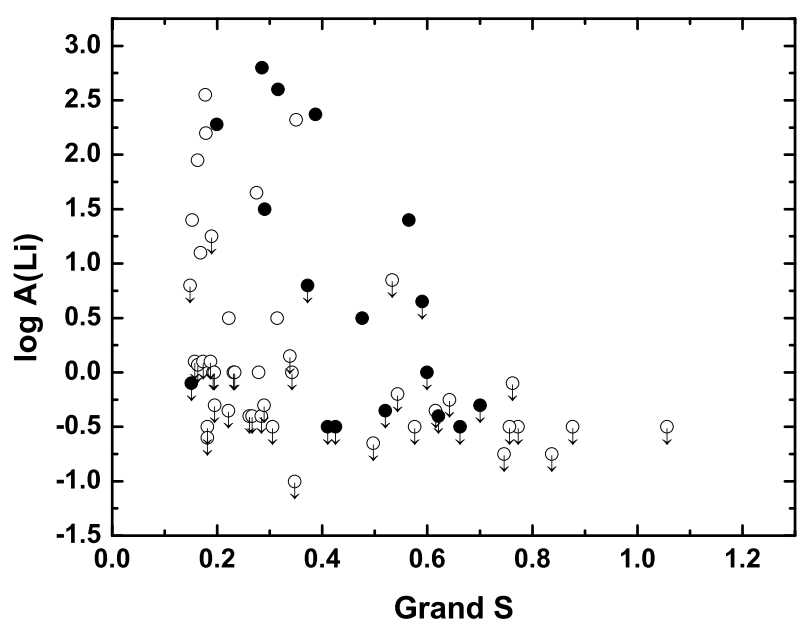

Fig. 10. The dependence of $\mathrm{Li}$ abundance on index of chromospheric activity $\mathrm{S}$, the notation is the same as in Fig. 8.

abundances of $\mathrm{Li}(\log A(\mathrm{Li})>2$, HD 63433, HD 130948, HD 190406, HD 217813) have higher $T_{\text {eff }}(5693$ to $5943 \mathrm{~K})$ than active stars with a low value of lithium $(\log A(\mathrm{Li})<1.5$, HD 59747, HD 111395, HD 141272), which have their $T_{\text {eff }}$ ranging from 5126 to $5648 \mathrm{~K}$. We can also observe that the stars with a determination of GrandS greater than 0.6 have lithium at the limit of detection. This would confirm the anticorrelation; however, the low number of stars does not allow us to generalise this conclusion. Moreover, since our spectra and those used to compute the $\mathrm{S}$ index were observed at different times, a reliable dependance between $v \sin i$ and chromospheric activity may not be observable.
Table 6 presents the parameters of the stars showing appreciable spectral peculiarities, first of all $\mathrm{H}_{\alpha}$ and $\mathrm{H}_{\beta}$ emissions. These stars show a high rotational velocity $v \sin i$, a value of $\sigma T_{\text {eff }}$ close to the average one, and a higher microturbulent velocity $V_{\mathrm{t}}$ than the average value. However, the star V833 Tau with the maximal emission has low values for all the parameters mentioned above and there is no line of lithium in its spectrum.

\section{Conclusion}

Physical parameters and chemical compositions were determined homogeneously for a large set of cool stars including both active and nonactive stars that could be compared. We found that

1. The general trends of abundance ratios with metallicity are consistent with previous results.

2. The behaviour of the abundances of the majority of elements in active and nonactive stars do not differ.

3. The Li is reliably detected in $54 \%$ of active stars, whereas only in $20 \%$ of other stars.

4. Our study of active stars has shown that there is no clear correlation between different activity indicators: $v \sin i, \mathrm{H}$ and $\mathrm{K}$ Ca II and $\mathrm{H}_{\alpha}, \mathrm{H}_{\beta}$ emissions, $\log A(\mathrm{Li})$. In particular, stars with high lithium abundances have different rotational velocities and show various degrees of activity in the calcium and hydrogen lines.

Acknowledgements. T.M. thanks the Laboratoire d'Astrophysique de Bordeaux for hospitality during this project. This research made use of the SIMBAD database, operated at the CDS, Strasbourg, France. It is based on data from the ESA Hipparcos satellite (Hipparcos catalogue). We also thank the referee for useful comments.

\section{References}

Alekseev, I. Y. 2006, Astrofiz., 49, 303

Alonso, A., Arribas, S., \& Martinez-Roger, C. 1996, A\&A, 313, 873

Benz, W., \& Mayor, M. 1984, A\&A, 138, 183

Biazzo, K., Frasca, A., Henry, G. W., et al. 2007, ApJ, 656, 474

Bienaymé, O., Soubiran, C., Mishenina, T., et al. 2006, A\&A, 446, 933

Blackwell, D. E., \& Lynas-Gray, A. E. 1998, A\&AS, 129, 505

Carlsson, M. 1986, Uppsala Obs. Rep., 33

Cayrel de Strobel, G. 2001, A\&A, 373, 159

Chugainov, P. F. 1966, Inform. Bull. Var. Stars., 122

Chugainov, P. F. 1991, Angular Momentum Evolution of Young Stars. Proceedings of the NATO Workshop, Sicily, Italy, Sep. 17-21 1990, ed. S. Catalano, \& J. R. Stauffer, 175

ESA 1997, The HIPPARCOS and TYCHO catalogues (Noordwijk, Netherlands: ESA Publications Division)

Favata, F., Micela, G., \& Sciortino, S. 1997, A\&A, 323, 809

Flower, P. J. 1996, ApJ, 469, 355

Fuhrmann, K. 2008, MNRAS, 384, 173

Gaidos, E. J., \& Gonzalez, G. 2002, New A, 7, 211

Gaidos, E. J., Henry, G. W., \& Henry, S. M. 2000, AJ, 120, 1006

Galazutdinov, G. A. 1992, Preprint SAO RAS, n92

Gershberg, R. E. 2002, Solar type activity of Main Sequence stars (Odessa: Astroprint) 
Glebocki, R., Gnacinski, P., \& Stawikowski, A. 2000, Acta Astron., 50, 509 Hall, J. C., Lockwood, G. W., \& Skiff, B. A. 2007, AJ, 133, 862

Høg, E., Fabricius, C., Makarov, V. V., et al. 2000, A\&A, 363, 385

Jasniewicz, G., Recio-Blanco, A., de Laverny, P., Parthasarathy, M., \& de Medeiros, J. R. 2006, A\&A, 453, 717

Katz, D., Soubiran, C., Cayrel, R., et al. 1998, A\&A, 338, 151

Kazarovets, A. V., Samus, N. N., Durlevich, O. V., et al. 1999, IBVS, N 4659

Korotin, S. A., \& Mishenina, T. V. 1999, AZh, 76, 611

Korotin, S. A., Andrievsky, S. M., \& Kostynchuk, L. Yu. 1999, Ap\&SS, 260, 531

Kovtyukh, V. V., \& Andrievsky, S. M. 1999, A\&A, 351, 597

Kovtyukh, V. V., Soubiran, C., \& Belik, S. I. 2004, A\&A, 427, 923

Kurucz, R. L. 1993, CD ROM n13

Luck, R. E., \& Heiter, U. 2006, AJ, 131, 3069

Lyra, W., \& Porto de Mello, G. F. 2005, Astron., A\&A, 431, 329

Masana, E., Jordi, C., \& Ribas, I. 2006, A\&A, 450, 735

Mishenina, T. V., \& Tsymbal, V. V. 1997, Pis'ma v Astron. Zh., 23, 693

Mishenina, T. V., Soubiran, C., Kovtyukh, V. V., \& Korotin, S. A. 2004, A\&A, 418,551
Mishenina, T. V., Bienaymé, O., Gorbaneva, T. I., et al. 2006, A\&A, 456, 1109 Nikityuk, T., \& Mishenina, T. 2006, A\&A, 456, 969

Nordström, B., Mayor, M., Andersen, J., et al. 2004, A\&A, 418, 989

Panagi, P. M., \& Mathioudakis, M. 1993, A\&AS, 100, 343

Queloz, D., Allain, S., Mermilliod, J.-C., Bouvier, J., \& Mayor, M. 1998, A\&A, 335,183

Randich, S., Gratton, R., Pallavicini, R., Pasquini, L., \& Carretta, E. 1999, A\&A, 348,487

Sakhibullin, N. A. 1987, AZh, 64, 1269

Soubiran, C., Bienaymé, O., Mishenina, T. V., \& Kovtyukh, V. V. 2008, A\&A, 480,91

Takeda, Y., \& Kavanomoto, S. 2005, PASJ, 57, 45

Takeda, Y., Kavanomoto, S., \& Honda, S., et al. 2007, A\&A, 468, 663

Tatischeff, V., Thibaud, J.-P., \& Ribas, I. 2008, [arXiv: 0801. 1777]

Tokovinin, A. A. 1992, A\&A, 256, 121

Tsymbal, V. V. 1996, ASP Conf. Ser., 108, 198

Valenti, J. A., \& Fischer, D. A. 2005, ApJS, 159, 141

Wright, J. T., Marcy, G. W., Buter, R. P., \& Vogt, S. S. 2004, ApJS, 152, 261 